\title{
Tele-health during COVID-19 Pandemic Lockdown in Pakistan - Experience of a Tertiary Care Hospital
}

\author{
Maaha Usmani ${ }^{1}$, Noureen Durrani ${ }^{1 *}$, Kiran Akhtar ${ }^{1}$, Mehreen Raza $^{1}$, Noman Ali ${ }^{1}$ and Faridah Amin ${ }^{1}$ \\ ${ }^{1}$ Liaquat National Hospital and Medical College, Karachi, Pakistan
}

\begin{abstract}
Introduction: COVID-19 (Coronavirus Disease 2019) caused by SARS-Cov-2 has quickly multiplied since its outbreak in humans. The utilization of tele-health came to the fore in this dire situation when it is absolutely necessary to maintain social distancing yet continue to get timely medical care. The current study is a preliminary audit of patients who consulted through the tele-health program in a tertiary care hospital during COVID-19 pandemic.

Methods: Through this study we share preliminary findings of the experience of a tertiary care hospital in Pakistan in terms of establishing and utilization pattern of the tele-health Program. The tele-health program was initiated by department of family medicine at Liaquat National Hospital (LNH), Karachi in collaboration with specialty of internal medicine, pulmonary medicine and infectious disease, approved by management and supported by outpatient, information technology, marketing and telecom services of LNH. The current study presents an audit of tele-health calls received during last week of March-April 2020. The data was obtained from structured clinical consultations notes recorded at the time of receiving patients' calls. Data was analyzed on SPSS version 20.

Results: Data of a total of 459 patients who availed tele-health services in March-April 2020 were entered and analyzed. The median no. of calls received were approximately 12 per day. Almost half of patients were between 18 to 39 years ( $n=224,48.40 \%) .407$ $(88.67 \%)$ consultations were initial while the rest were follow-up patients. Total 482 complaints were received including 9 requests for direct referrals which were directed to other specialized clinics. Respiratory complains were most frequently encountered (212, $46.19 \%)$. Among the 212 respiratory complaints, 103 (47.64\%) were COVID-19 related complaints. Out of 459 patients, only 7 (1.5\%) reported symptoms severe enough to be advised to visit hospital.

Conclusion: This audit summarizes the pattern of utilization of tele-health services during the initial lock-down phase of the pandemic. Basic medical advice to control symptoms may prevent patients presenting with complications and severe symptoms to tertiary care hospitals once social distancing protocols are relaxed. Acceptability of the idea by the patients, and common medical complains being dealt with ease with tele-health consultation rationalizes the need to continue and built up these services for all specialties.
\end{abstract}

Keywords: Tele-medicine, tele-health, COVID-19 pandemic, SARS-Cov-2, healthcare.

\section{INTRODUCTION}

COVID-19 (Coronavirus Disease 2019) caused by SARS-cov-2 has quickly multiplied since its outbreak in humans, affecting majority of the countries worldwide; and as of March $11^{\text {th }} 2020$, a pandemic was declared by World Health Organization [1].

The rate of morbidity and mortality has been alarmingly high relative to other flu-like illnesses since the start of out-break, compelling governments all over the world to take stringent measures to prevent the spread [2], limit the number of new cases and to treat the affected population.

As the situation progressed, the detrimental effects of the pandemic were quickly realized on the healthcare resources and the economy. In terms of prevention, the most reliable tool was 'social distancing [3] because as is the case with most of the infectious diseases; this virus also spreads through droplets [4]. Thus social distancing was rendered essential to prevent the exponential spread of the disease. This brought about

\footnotetext{
*Corresponding Author: Noureen Durrani, Liaquat National Hospital and Medical College, Karachi, Pakistan; Email: Noureen.Durrani@lnh.edu.pk Received: June 08, 2020; Revised: June 25, 2020; Accepted: July 01, 2020 DOI: https://doi.org/10.37184/lnjpc.2707-3521.1.26
}

the implementation of lock-down among countries [5] which indeed was the most important factor in controlling the disease and alleviation of the deadly situation at the peak of Pandemic across the globe. 'Stay at home, stay safe' has become the order of the day; only to be breached if absolutely necessary [3].

All the possible measures are being attempted to avoid community gatherings and congregations of every sort. This has brought about a revolution in itself in nearly every field of life, ranging from homeschooling and virtual education systems [6] to 'working from home' in offices, wherever possible. In the context of primary healthcare, it has brought about a need of effective and accessible healthcare through 'tele-health' which is based on provision of healthcare through utilization of information and communication by technology-based methods. It enables the clinicians, and health system to provide care to remote population without increasing the risk of cross-contamination. Tele-health though not routinely employed, is not a new concept in the field of medicine. Perhaps one of the most famous and initial use of hospital based telemedicine example comes to us from the closed-circuit television link between Nebraska psychiatric institute and Norfolk State hospital 
in the late 1950s and in the beginning of early 1960s [7]. The utilization of tele-health came to the fore in this dire situation when it is absolutely necessary to maintain social distancing. This is particularly useful while providing routine healthcare access to those at a higher risk of being affected, for example elderly population with pre-existing medical conditions without the risk of exposure in a congested hospital or hospital waiting rooms.

Advantages of tele-health also include facilitation of triage so that the front-liners are not overwhelmed [8]; leading to a more-focused approach in dealing with the acute emergencies. It is also cost-effective in the long run; safe-guarding the medical supplies for those in acute need. It also efficiently deals with the traffic problems, hurdles in reaching the hospital for non-acute consultations and long waiting hours. While tele-health seems to provide the answer to many of the challenges in healthcare practice during the current ongoing pandemic, it brings its own set of challenges. Practicing tele-health by individual clinicians is not a sustainable approach. It requires a whole systematic strategy involving operational tele-health networks, tele-health policies, procedures and technology infrastructure during routine practice; up scaling, should the need arise, in case of emergency situations like the current pandemic [9].

While tele-health has developed and grown exponentially during the past decade in the developed countries, yet Pakistan has not been able to obtain any significant benefit from this particular field of medicine [10]. Indeed, there are very few hospitals all over Pakistan with a definite set-up during the pandemic, let alone the routine clinical practice [11]. There is a dearth of clinicians and healthcare practitioners who are trained in this field to confidently put it in practice. Limitations also include the fact that many essential components of a live consultation cannot be performed remotely, for example, physical examinations and diagnostics (like imaging and cultures).
In the nutshell, tele-health can be the solution in the present times for making the healthcare accessible to urban and rural population with the omission of many hurdles and challenges. This would although need vigorous training, proper formulation of guidelines and a user and consumer-friendly system to work efficiently. The current study therefore presents a preliminary audit of patients who consulted through of tele-health program implemented in a tertiary care hospital during COVID-19 pandemic.

\section{METHODOLOGY}

The current study presents the experience of a tertiary care hospital in Pakistan in terms of program utilization pattern and patients' clinical complaints that were received in telehealth Program. The tele-health program was initiated by Family Medicine Department at Liaquat National Hospital, Karachi in collaboration with Department of Pulmonology, Internal Medicine and Infectious Diseases, supported by LNH Outpatient Department (OPD), Information Technology (IT), Tele-communication and Marketing team of the hospital. The program was implemented following the decision of nationwide lockdown by Government of Pakistan on March $25^{\text {th }}, 2020$. The lockdown in Pakistan started from Karachi, where first COVID-19 case was detected. Therefore, keeping in view the utmost need of time, the proposal of the tele-health program was forwarded to the Institutional Management for approval and hence program was implemented within 2 weeks of lockdown. Core team to plan, implement and assure the quality of the program consisted of a Family Physician, OPD manager, Tele-communication expert, Marketing manager and IT personnel. A team of five members were trained to run the service which included telephone operator (OPD assistants), IT personnel, nurses and doctors. Each of these team members were posted for 2 hours/day to respond to patients' calls. Video and telephone calls were attended by family physicians, internists, pulmonologists or infectious disease (ID) specialists according to patient's complain. A flow chart of the process which was established to receive, answer and resolve the patients' complaints is depicted in Fig. (1).

\begin{tabular}{|c|c|c|}
\hline $\begin{array}{l}\text { Link for video conferencing on } \\
\text { LNH website }\end{array}$ & $\begin{array}{l}\text { Phone call on Hot-line numbers } \\
\text { attended by OPD assistants }\end{array}$ & OPD assistant to attend follow up call \\
\hline$\frac{1}{t}$ & $\frac{1}{t}$ & 4 \\
\hline $\begin{array}{l}\text { Patient registered his name, phone } \\
\text { number, city and age on web link }\end{array}$ & \begin{tabular}{|l|} 
Patient's name, phone number, city and age \\
registered by call attendant (annexure $2 \mathrm{a})$.
\end{tabular} & $\begin{array}{l}\text { In case of referral to OPD/ER, call } \\
\text { was reverted back to registration }\end{array}$ \\
\hline$\downarrow$ & $\frac{1}{1}$ & deskroom for referral guidance \\
\hline Video call forwarded to doctor 1 & Telephone call forwarded to doctor 2 & 4 \\
\hline$\downarrow$ & $\downarrow$ & \multirow{2}{*}{\begin{tabular}{|c|}
$\begin{array}{c}\text { Copy of documented notes and patients' } \\
\text { complaints forwarded to ITdepartment } \\
\text { for transcription }\end{array}$ \\
\end{tabular}} \\
\hline \multicolumn{2}{|c|}{$\begin{array}{c}\text { Doctor provided consultation and noted down patient's } \\
\text { complaint and management }\end{array}$} & \\
\hline$\checkmark$ & $\frac{1}{t}$ & 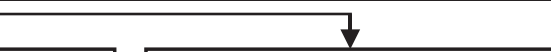 \\
\hline $\begin{array}{l}\text { Advised follow-up } \\
\text { through audio call }\end{array}$ & $\begin{array}{l}\text { erred to other } \\
\text { specialty }\end{array}$ & $\begin{array}{l}\text { Treatment given and } \\
\text { complaint resolved }\end{array}$ \\
\hline
\end{tabular}

Fig. (1): Process flow for tele-health program. 
This audit includes the preliminary data of patients' calls received during March-April 2020. Data was obtained from structured clinical consultation notes recorded at the time of receiving patients' call. The obtained data was entered into IBM SPSS (version 20) for statistical analysis. Frequencies and percentages were computed to report categorical variables such as patients' complaints, gender and type of consultation. Median and interquartile range was computed to express continuous variables like participants' age and number of calls per day. Chi-square/Fisher-exact test was applied to assess the significant difference in patients' age, gender and type of consultation with patients' complaints. P-value $<0.05$ was taken as statistically significant.

\section{RESULTS}

A total of 459 patients approached tele-health program during last week of March-2020 till April-2020. Out of 459 calls, $422(91.39 \%)$ were audio calls and remaining $(n=37,8.06 \%)$ were video calls. The median no. of calls/day was approximately 12 . Most of the calls were received from Sindh $(n=407,88.67 \%)$ followed by Punjab ( $n=25,7.63 \%)$, Baluchistan $(n=10,2.18 \%)$, Khyber Pakhtunkhwa $(n=6,1.31 \%)$ and Gilgit $(n=1$, $0.22 \%$ ). Calls were received from 19 different cities of the country and majority of the calls were received from Karachi $(n=385,83.87 \%)$. The age range of patients was infants to 92 years. Almost half of patients were between 18 to 39 years $(n=224,48.40 \%) .63(13.73 \%)$ patients were aged $<12$ years. $92(20.04 \%)$ patients were in age group of $40-60$ years. $55(11.98 \%)$ patients were older than 60 years. Out of 459 calls, $407(88.67 \%)$ consulted for the first time while $52(11.32 \%)$ calls were made by patients who were advised for follow-up visit before COVID-19 pandemic.
Total 482 complaints were received including 9 requests which needed direct referral to a specialist. The most frequent complaints were regarding respiratory symptoms (212, 46.19\%). Among 212 respiratory complaints, $103(47.64 \%)$ were COVID-19 related queries. This was followed by gastrointestinal (GI) complains ( $n=55$, $11.98 \%)$ among which acute gastroenteritis $(n=26$, $47.27 \%$ ) was the most frequently reported complain. Among musculoskeletal symptoms ( $n=31,6.75 \%)$, nonspecific muscle pain was the most common complain $(n=14,45.16 \%)$. Obstetrics \& gynecology issues were reported by a few patients $(n=28,6.10 \%)$, majority were regarding antenatal care $(n=13,46.43 \%)$. Stress and anxiety composed most of the psychological concerns $(n=27,5.88 \%)$, febrile illness ranked the highest $(n=15$, $68.18 \%)$ among symptoms of infectious diseases $(n=22$, $4.79 \%)$. Among neurological complains (21, 4.58\%) headache was the most reported complain $(n=10$, $47.62 \%)$. Diabetic control $(n=10,55.55 \%)$ was the most common concern among patients consulting for endocrine-related medical issues $(n=18,3.92 \%)$. Among problems regarding ear, nose, throat (ENT), complaints which were frequently reported were sore throat $(n=16,3.49 \%)$ and symptoms of allergic rhinitis $(n=5$, $31.25 \%)$ respectively. Among renal symptoms ( $n=12$, $2.61 \%$ ), burning micturition and increased frequency of urination $(n=8,66.67 \%)$ were common complains. 11 consulted for skin problems $(n=11,2.40 \%)$ while 20 patients presented with miscellaneous symptoms $(20$, $4.36 \%)$. Out of these $20(4.36 \%), 6(30 \%)$ were related to cardiovascular diseases, 5 (25\%) were surgery related complaints, 5 (25\%) were eye symptoms, 2 (10\%) were related to oncology, $1(5 \%)$ was regarding the use of certain medicines, while 1 (5\%) was dental. 9 (1.96\%) patients were directed to other specialty based on need.

Table 1: Break-up of patients' complaints received according to their age, gender and type of consultation.

\begin{tabular}{|c|c|c|c|c|c|c|c|c|c|c|c|c|}
\hline & $\begin{array}{c}\text { A } \\
\mathrm{n}(\%)\end{array}$ & $\begin{array}{c}\text { B } \\
\text { n (\%) }\end{array}$ & $\begin{array}{c}\mathrm{C} \\
\mathrm{n}(\%)\end{array}$ & $\begin{array}{c}\text { D } \\
\text { n (\%) }\end{array}$ & $\begin{array}{c}E \\
n(\%)\end{array}$ & $\begin{array}{c}F \\
n(\%)\end{array}$ & $\begin{array}{c}\mathbf{G} \\
\mathrm{n}(\%)\end{array}$ & $\begin{array}{c}\mathrm{H} \\
\mathrm{n}(\%)\end{array}$ & $\begin{array}{c}\text { I } \\
\text { n (\%) }\end{array}$ & $\begin{array}{c}\mathrm{J} \\
\mathrm{n}(\%)\end{array}$ & $\begin{array}{c}\mathrm{K} \\
\mathrm{n}(\%)\end{array}$ & $\begin{array}{c}\mathrm{L} \\
\mathrm{n}(\%)\end{array}$ \\
\hline \multicolumn{13}{|c|}{ Age (in years) } \\
\hline$<12$ & $4(6.3)$ & $18(28.6)$ & $24(38.1)$ & $0(0)$ & $1(1.6)$ & $0(0)$ & $7(11.1)$ & $1(1.6)$ & $0(0)$ & $2(3.2)$ & $5(7.9)$ & $2(3.2)$ \\
\hline $12-17$ & $1(9.1)$ & $1(9.1)$ & $5(45.5)$ & $1(9.1)$ & $0(0)$ & $0(0)$ & $2(18.2)$ & $0(0)$ & $0(0)$ & $2(18.2)$ & $1(9.1)$ & $0(0)$ \\
\hline $18-39$ & $5(2.2)$ & $18(8.0)$ & 111(49.6) & $12(5.4)$ & $9(4)$ & $12(5.4)$ & $8(3.6)$ & $6(2.7)$ & $25(10.5)$ & $4(1.8)$ & $13(5.8)$ & $6(2.7)$ \\
\hline $40-60$ & $2(1.9)$ & $13(12.3)$ & $47(44.3)$ & $4(3.8)$ & $5(4.7)$ & $16(15.1)$ & $7(6.6)$ & $1(0.9)$ & $3(3.3)$ & $2(1.9)$ & $3(2.8)$ & $5(4.7)$ \\
\hline$>60$ & $4(7.3)$ & $5(9.1)$ & $25(45.5)$ & $4(7.3)$ & $3(5.5)$ & $3(5.5)$ & $3(5.5)$ & $4(7.3)$ & $0(0)$ & $1(1.8)$ & $0(0)$ & $1(1.8)$ \\
\hline p-value & 0.084 & ${ }^{* *}<0.001$ & 0.585 & 10.158 & t0.813 & $t^{* \star} 0.002$ & t0.058 & +0.224 & $t^{* \star} 0.001$ & t0.076 & †0.119 & - \\
\hline \multicolumn{13}{|l|}{ Gender } \\
\hline Female & $7(3.1)$ & $32(14.2)$ & $77(34.2)$ & $13(5.8)$ & $10(4.4)$ & $18(8)$ & $16(7.1)$ & $8(3.6)$ & $28(100)$ & $4(1.8)$ & $7(3.1)$ & $6(2.7)$ \\
\hline Male & $9(3.8)$ & $23(9.8)$ & 135(57.7) & $8(3.4)$ & $8(3.4)$ & $13(5.6)$ & $11(4.7)$ & $4(1.7)$ & $0(0)$ & $7(3)$ & $15(6.4)$ & $8(3.4)$ \\
\hline$p$-value & 0.668 & 0.147 & ${ }^{* *}<0.001$ & 0.227 & 0.571 & 0.297 & 0.273 & 0.215 & - & 0.395 & 0.098 & - \\
\hline \multicolumn{13}{|c|}{ Type of Consultation } \\
\hline Initial & $14(3.4)$ & $47(11.5)$ & 196(48.2) & $19(4.7)$ & $12(2.9)$ & $26(6.4)$ & $26(6.4)$ & $9(2.2)$ & $23(5.7)$ & $10(2.5)$ & $21(5.2)$ & $12(2.9)$ \\
\hline Follow-up & $2(3.8)$ & $8(15.4)$ & $16(30.8)$ & $2(3.8)$ & $6(11.5)$ & $5(9.6)$ & $1(1.9)$ & $3(5.8)$ & $5(9.6)$ & $1(1.9)$ & $1(1.9)$ & $2(3.8)$ \\
\hline p-value & 0.7 & 0.422 & ${ }^{*} 0.018$ & +1.00 & $t^{\star} 0.010$ & +0.377 & 10.343 & t0.145 & +0.231 & +1.00 & 10.494 & - \\
\hline
\end{tabular}

A:otolaryngology, B:gastroenterology, C:respiratory, D:neurology, E:endocrinology, F:Rheumatology, G:psychology, H:nephrology, I:obstetrics \& gynecology, J:dermatology, K:infectious diseases, L:others

${ }^{\dagger}$ Fisher-Exact test was reported, ${ }^{*} p<0.05,{ }^{* *} p<0.01$ 
The proportion of male patients $(n=135,57.7 \%)$ who presented with respiratory complaints was significantly higher as compared to female patients ( $n=77,34.2 \%)$ $(p<0.001)$. The frequency of respiratory complaints was significantly high for initial consultation $(n=196$, $48.2 \%)$ as compared to follow-up consultations $(n=16$, $30.8 \%) \quad(p=0.018)$. GI complaints were significantly higher among patients $<12$ years of age $(n=18,28.6 \%)$ $(p<0.001)$. Middle aged group (40-60 years) $(n=16$, 15.1) significantly reported higher rheumatology/ musculoskeletal complaints as compared to other people $(p=0.002)$. Obstetric and gynecological issues were more likely to be reported by young females 18-39 years of age $(n=27,10.3)(p<0.001)$ (Table 1).

The management of patients on tele-health calls is depicted in Fig. (2). Almost all of the patients' issues were managed on call $(n=452,98.47 \%)$ while $7(1.5 \%)$ were advised to visit hospital for consultation due to ambiguity or severity of complains. Out of 452, $273(59.5 \%)$ were prescribed a medicine. $70(15.3 \%)$ didn't require any treatment and they were counseled accordingly. $44(9.6 \%)$ patients were referred to other specialties. $8(1.7 \%)$ were advised for investigations. 57 (12.4\%) queries were regarding general information regarding clinic timing etc. which were resolved immediately.

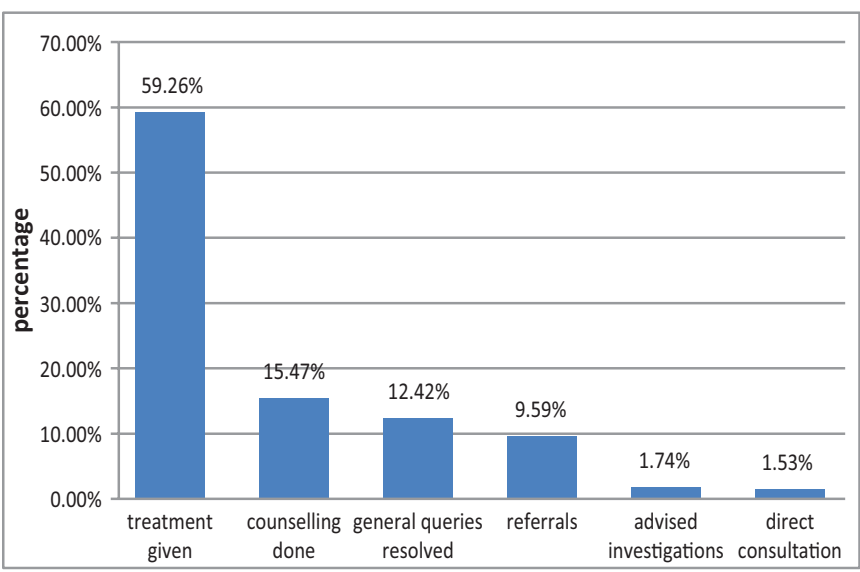

Fig. (2): Management of patients consulting doctors through tele-health program.

Regarding management, out of 273 patients who were advised treatment besides counseling, steam inhalation, gargles, paracetamol, honey water and vitamin $\mathrm{C}$ were advised for majority of patients ( $n=79,17.2 \%)$. Analgesia was advised to $32(6.7 \%)$ patients. Anti-histamines were given to $25(5.4 \%)$ patients. $23(8.4 \%)$ patients were advised to follow their previous prescriptions with minor alterations. $19(7 \%)$ were prescribed paracetamol alone. Oral rehydration therapy was prescribed to 15 (3.3\%) patients, including 7 who were also prescribed probiotics. The number of patients that were prescribed antibiotics (oral and local) were 15 (3.3\%). 11 (2.4\%) cases were prescribed a proton pump inhibitors (PPI) while antacids were given to $4(0.9 \%)$ patients. Specific preventive measures were advised in $9(1.9 \%)$ cases.
$8(1.7 \%)$ patients were prescribed antenatal nutritional supplements. $6(1.3 \%)$ patients were prescribed with inhalers and nebulizers $5(1.7 \%)$ patients presented with multiple urinary complains like burning, frequency, decreased quantity of urine and were advised hydration, and conservative management of symptoms. Eye lubrication was advised in $4(0.9 \%)$ cases. Thyroxine adjustment was done in $4(0.9 \%)$ hypothyroid patients. 4 $(0.6 \%)$ patients needed lifestyle modification exclusively and were guided accordingly. $4(0.9 \%)$ patients presented with malaria, vertigo, sleep problems, ear pain; and were respectively given anti-malarial, histamine analogue, advice regarding sleep hygiene and ear drops. The use of anti-hemantic medicines (iron supplements) was advised to $1(0.2 \%)$ patient only. $2(0.4 \%)$ of the patients with skin rash were advised a topical emollient, along with symptomatic management. $2(0.4 \%)$ patients complained of oral ulcers, and were prescribed PPI, mouthwash and folic acid. $1(0.2 \%)$ patient was directed to have a home health visit.

\section{DISCUSSION}

'Social distancing' reduces the person-to-person contact, thereby plays the cornerstone role in the prevention of viral transmission [12]. Therefore, we believe that during COVID-19 pandemic, nationwide lockdown and closure of primary care services and outpatient departments of hospitals, tele-health clinics were an ideal substitute to direct consultations and provided the answer to many acute healthcare problems of the masses in remote areas, as well as to those unable to reach the hospital. This is supported by the number and variety of cases consulted through our tele-health service, while effectively reducing waiting time and added costs incurred both upon the community and the healthcare system[13]. Moreover, it was also beneficial in protecting the healthcare community from unnecessary exposure, which may result in infecting other patients and healthcare professionals, thereby enlarging the gap of availability of doctors and healthcare staff during the crucial times of their need [14]. Recently, Duffy and Lee emphasized that direct consultations should become second, third or even last option while catering to the needs of the patients, keeping in view the patients' priorities for inexpensive and convenient care [15].

Tele-health can take mainly one of three forms; namely, video, audio telephone calls and instant messaging in the form of electronic mails, health system messaging services and the use of mobile applications to track patient recorded metrics. While video consultations provide an added benefit of viewing the patient and performing a certain range of examination, each of these types can be beneficial as well easily accessible by the patient [16]. This study aims to present the results of a real time Tele-health program, implemented to provide primary healthcare to patients of all age groups during the COVID-19 pandemic after the execution of nationwide lockdown. Tele-health owns a number of key 
strengths that can enhance an emergency response in the setting of an environmental or biological hazards. During infectious disease outbreaks, tele-health can make it possible to perform remote triaging of care and provide rapidly accessible information through technology - such as chatbots, as seen in Singapore during COVID-19 [17].

It has been documented in literature that tele-health has been efficiently implemented for diabetic care [18], intensive care management [19] and pediatric care [20]. In our current study we received complaints related to respiratory system (46.19\%), GI system (11.98\%), rheumatology $(6.75 \%)$, obstetrics \& gynecology $(6.10 \%)$, psychological concerns $(5.88 \%)$, infectious diseases $(4.79 \%)$, neurology $(4.58 \%)$, endocrinology (3.92\%), otolaryngology (3.49\%), nephrology $(2.61 \%)$, dermatology $(2.40 \%)$, cardiovascular disease $(1.31 \%)$, surgery $(1.09 \%)$, ophthalmology (1.09\%), oncology $(0.44 \%)$, hematology $(0.22 \%)$, and dental diseases $(0.22 \%)$.

During the entire period of the study, total 459 calls were received, among which $88.67 \%$ were new patients who consulted for the first time in our institute. The higher proportion of new patient registrations clearly indicates that people in our local setting accepted the tele-health program, and they considered it a feasible way of consulting the medical experts for their primary care during COVID-19 pandemic and nationwide lockdown. In 2017, a pilot study was conducted at Brodstone Hospital, Nebrasaka [21] with the aim of finding the response of tele-health for initial evaluation of general surgery patients. In the study, mobile phone pictures were used for early detection of post-operative complications following appendectomy. This method was found to be $100 \%$ sensitive, $91.6 \%$ specific, with a negative predictive value of 100 . Matawalli and Ibrahim also pointed out that interactive tele-health services (which include phone conversations, online communication and home visits) provide real-time interactions between patient and provider [22].

Simply, tele-health is not just an appropriate alternative to in-person patient consultation during the pandemic but it has also proved to be effective in managing patients in routine care under normal circumstances and making necessary clinical decisions while saving the time and expense of direct consultation.

In the current study, out of $\mathbf{4 5 9}$ patients who registered and contacted our tele-health program, only $1.7 \%$ people were advised to visit the hospital while $98.47 \%$ of them were managed on audio call. In addition to this, $46.19 \%(n=212)$ of the received complaints were related to respiratory system with significantly higher proportion of males. Out of 212 respiratory complaints, 103 (47.64\%) were COVID-19 related complaints. Even after ignoring the number of COVID-19 complaints, the respiratory issues still stand as the commonest patient complaint for which patient seek telephonic consultation. The findings undoubtedly suggest that, during the pandemic people became more vigilant even for minor respiratory issues and attempted to visit doctors which would not only have increased the burden on healthcare system but would have also exposed them to a higher risk of acquiring the COVID-19 infection. Therefore, the implementation of tele-health program in our setting was not only an effective measure in meeting the needs of the community but was also accepted by our local population. It also reflected positively upon the importance of telemedicine in a developing country with shortage of resources, and a dearth of healthcare facilities available to the community, including a disparity of quality of care between urban and rural population and the number of qualified physicians catering to the need of target population [23]. Although this is a nascent introduction in our current practice, yet a turnout of 459 patients over the period of 1.5 months shows promise towards future development. This also implies that with more training, advancement of the infrastructure, and better collaboration of all the concerned departments as well as awareness among targeted population it could be developed into a routine care practice.

Therefore, we recommend the utilization of tele-health to be developed, and improved upon, with a more focused emphasis upon training of the healthcare personnel. The training must focus upon the facilitation of patients keeping in view the limitations of a virtual consultation.

The take home message is that tele-health services can provide a substitute for walk-in clinic during unfavorable times along with supplying a scope of multiple possibilities that can be worked upon to provide a costeffective healthcare to the people with reduced burden upon the economy. It requires the implementation of a uniform national/regional policy in this regard.

\section{CONCLUSION}

This audit summarizes the pattern of utilization of telehealth services during the initial lock-down phase of the pandemic. Acceptability of patients and common medical complains being dealt with ease and minimum drug intervention rationalizes the need to continue and built up these services for all specialties. Basic medical advice to control symptoms may prevent patients presenting with complications and severe symptoms to tertiary care hospitals once social distancing protocols are relaxed. This added burden may pose a threat to an already exhausted healthcare system with COVID patients. Tele-health can provide the bridging solution, enabling people to access care from the comfort of their homes. It would be worthwhile to study long term advantages of the tele-health program on the primary healthcare of the population.

\section{CONFLICT OF INTEREST}

The authors declare no conflict of interest. 


\section{ACKNOWLEDGEMENTS}

We acknowledge Administrative Officer, Ms. Salima Mehdi for providing technical assistance for the program and helping with data entry.

\section{REFERENCES}

1. Jin $\mathrm{Y}$, Yang $\mathrm{H}$, Ji W, Wu W, Chen $\mathrm{S}$, Zhang $\mathrm{W}$, et al. Virology, epidemiology, pathogenesis, and control of COVID-19. Viruses 2020; 12(4): 372.

2. Triggle CR, Bansal D, Farag EABA, Ding H, Sultan AA. COVID-19: learning from lessons to guide treatment and prevention interventions. mSphere 2020; 5(3): e00317-20.

3. Wilder-Smith A, Freedman DO. Isolation, quarantine, social distancing and community containment: pivotal role for old-style public health measures in the novel coronavirus (2019-nCoV) outbreak. J Travel Med 2020; 27(2): taaa020.

4. Güner R, Hasanoğlu I, Aktaş F. COVID-19: prevention and control measures in community. Turk J Med Sci 2020; 50(SI-1): 571-7.

5. Waris A, Atta UK, Ali M, Asmat A, Baset A. COVID-19 outbreak: current scenario of Pakistan. New Microbes New Infect 2020; 35: 100681.

6. Joseph C, Kerryn B-H, Rudolph J, Malkawi B, Glowatz M, Burton $\mathrm{R}$, et al. COVID-19: 20 countries' higher education intra-period digital pedagogy responses. J Appl Learn Teach 2020; 1: 1-20.

7. Board on Health Care Services; Institute of Medicine. The role of telehealth in an evolving health care environment: workshop summary. Available from: https://www.ncbi.nlm.nih.gov/books/ NBK207145/. Washington (DC): National Academies Press; 2012.

8. Lurie N, Carr BG. The role of telehealth in the medical response to disasters. JAMA Intern Med 2018; 178: 745-6.

9. Smith AC, Thomas E, Snoswell CL, Haydon H, Mehrotra A, Clemensen J, et al. Telehealth for global emergencies: implications for coronavirus disease 2019 (COVID-19). J Telemed Telecare 2020; 26(5): 309-13.

10. Ahmed A, Ahmed M. The telemedicine landscape in Pakistan-why are we falling behind? J Pak Med Assoc 2018; 68(12): 1820-2.

11. These are the $7 \mathrm{WHO}$ designated coronavirus test centers in Pakistan. https://propakistani.pk/2020/02/28/these-are-the-7-who- designated-coronavirus-test-centers-in-pakistan/. (Accessed on: May 31, 2020).

12. Public Health England. Coronavirus (COVID-19): what is social distancing? Available from: https://publichealthmatters.blog.gov. uk/2020/03/04/coronavirus-covid-19-what-is-social-distancing. (Accessed on: May 31, 2020).

13. Caffery LJ, Farjian, M, Smith AC. Telehealth interventions for reducing waiting lists and waiting times for specialist outpatient services: a scoping review. J Telemed Telecare 2016; 22(8): 50412.

14. Wax RS, Christian MD. Practical recommendations for critical care and anesthesiology teams caring for novel coronavirus (2019nCoV) patients. Can J Anaesth 2020; 67: 568-76.

15. Duffy S, Lee TH. In-person health care as option B. N Engl J Med 2018; 378(2): 104-6.

16. Hakim AA, Kellish AS, Atabek U, Spitz FR, Hong YK. Implications for the use of telehealth in surgical patients during the COVID-19 pandemic. Am J Surg 2020; S0002-9610(20): 30231-2.

17. Singapore government launches COVID-19 chatbot. Available from: https://www.opengovasia.com/singapore-governmentlaunches-covid-19-chatbot/. (Accessed on: May 28, 2020).

18. Lee JY, Lee SWH. Telemedicine cost-effectiveness for diabetes management: a systematic review. Diabetes Technol Ther 2018; 20(7): 492-500.

19. Fortis S, Sarrazin MV, Beck BF, Panos RJ, Reisinger HS. ICU telemedicine reduces interhospital ICU transfers in the veterans health administration. Chest 2018; 154(1): 69-76.

20. Sheikhtaheri A, Kermani F. Telemedicine in diagnosis, treatment and management of diseases in children. Stud Health Technol Inform 2018; 248: 148-55.

21. Schroeder C. Pilot study of telemedicine for the initial evaluation of general surgery patients in the clinic and hospitalized settings. Surg Open Sci 2019; 1(2): 97-9.

22. Geidam MA, Bello IA, Prasad R. Health care using telemedicine: a case study of Yobe State, Nigeria. Int J Comput Sci Netw 2014; 3(5): 385-92.

23. Ullah N, Khan P, Sultana N, Kwak KS. A telemedicine network model for health applications in Pakistan: current status and future prospects. Int J Digit Content Technol Appl 2009; 3(3): 149-55. 